\title{
When and Why to Replicate: As Easy as 1, 2, 3?
}

\section{Sarahanne M. Field, Rink Hoekstra, Laura Bringmann and Don van Ravenzwaaij Rijksuniversiteit Groningen}

Word count:

Corresponding author: Sarahanne M. Field

University of Groningen, Department of Psychometrics and Statistics

E-mail should be sent to sarahanne.field@gmail.com

\begin{abstract}
The crisis of confidence in psychology has prompted vigorous and persistent debate in the scientific community concerning the veracity of the findings of psychological experiments. This discussion has led to changes in psychology's approach to research, and several new initiatives have been developed, many with the aim of improving our findings. One key advancement is the marked increase in the number of replication studies conducted. We argue that while it is important to conduct replications as part of regular research protocol, it is neither efficient nor useful to replicate results at random. We recommend adopting a methodical approach toward the selection of replication targets to maximize the impact of the outcomes of those replications, and minimize waste of scarce resources. In the current study, we demonstrate how a Bayesian re-analysis of existing research findings followed by a simple qualitative assessment process can drive the selection of the best candidate article for replication. The files associated with this article can be found on the project's OSF page: https://doi.org/10.17605/OSF.IO/3RF8B.
\end{abstract}

Keywords: replication, transparency, psychological science, Bayesian reanalysis.

"The case against science is straightforward: much of the scientific literature, perhaps half, may simply be untrue. Afflicted by studies with small sample sizes, tiny effects, invalid exploratory analyses, and flagrant conflicts of interest, together with an obsession for pursuing fashionable trends of dubious importance, science has taken a turn towards darkness." (Horton, 2015)

As dramatic as Horton's claims may sound, the concerns he raises are not without merit. They reflect the discussions around the 'crisis of confidence' in psychology (Pashler 
\& Wagenmakers, 2012), which has become a popular topic due, in large part, to the low rate at which published studies can be replicated. which has been reported in psychological research. Prior to the crisis of confidence breaking into the collective awareness of the field of psychology, Ioannidis published a now well-cited theoretical article (2005), in which he argued that more than half of published findings may be false. The landmark mass replication effort of the Open Science Collaboration (henceforth OSC; 2015) gave empirical support for Ioannidis' claims a decade after they were made, but reported an even more bleak narrative. Only $36 \%$ of replication studies were successful in yielding a result comparable to that reported in the original article. To put this finding in context: had all of the original results been true, a minimum reproducibility rate of $89 \%$ would be expected, according to the OSC (2015). This reproducibility crisis is not only the preserve of psychology: reports of poor reproducibility have come from other sources in the scientific community (Baker, 2016; Begley \& Ioannidis, 2015; Chang \& Li, 2018).

The literature has suggested a number of potential causes for poor reproducibility of research findings. One of the most obvious candidates is the publish or perish culture in academia (Grimes, Bauch, \& Ioannidis, 2018), which describes the pressure on researchers to publish much and often in order to maintain their university faculty positions, or to move up the hierarchical 'ladder'. Another is the over-reliance of researchers on null hypothesis significance testing, or NHST (Fidler \& Wilcox, 2018; Szucs \& Ioannidis, 2017). Fisher had only intended the $p$-value to be a rough guide to interpreting evidence against the null; for small $p$-values to be taken as nothing more than an indication that a given finding warranted more investigation (Goodman, 2008). Despite this, researchers often assume that a single significant $p$-value translates into scientific fact, which places huge importance on the finding of a significant $p$-value. Additionally, it is supposed that the ubiquity of NHST contributes to publication bias, which in turn leads to poor reproducibility (Fidler \& Wilcox, 2018). Combined with the problematic publish or perish culture, and one reaches a plausible explanation of why questionable research practices (QRPs) are common in psychology (John, Loewenstein, \& Prelec, 2012).

QRP refers to a number of practices which lead to poor quality research, and shaky conclusions. HARKing (hypothesizing after the results are known), p-hacking (where one massages the data to procure a significant $p$-value) and the 'file drawer' problem (where researchers do not attempt to publish their null results) are all examples of QRPs (Kerr, 1998; John et al., 2012; Rosenthal, 1979). These lead to a literature that is unreliable, and apparently in many cases (and often as a result), impossible to replicate. Another QRP involves unadjusted sequential testing, whereby an individual evaluates the $p$-value as the data come in, collecting more data until some threshold is reached (usually this threshold is 'statistical significance' with a fixed alpha of .05, but see Benjamin et al., 2018), and failing to adjust alpha to accommodate for the multiple testing iterations taken. This QRP leads to a large discrepancy between nominal and actual alpha in published studies, and is responsible for the overestimation of the importance of many study results.

Irrespective of the causes of the crisis of confidence we face, its consequence is irrefutable. Scientific and lay communities alike have lost faith in the veracity of many of psychology's key findings, and are hesitant to trust the conclusions upon which they are based. A recent online Nature news story suggested that most scientific results shouldn't be trusted (Baker, 2015). Psychology has been called 'broken' (Woolston, 2015); others have 
referred to the "terrifying unraveling" of the field (Woolston, 2016). The push for resolving the crisis situation has acted as the catalyst for the start of many initiatives, conceived in the spirit of improving reproducibility. A recent example is the peer reviewers' openness initiative (Morey et al., 2016), in which reviewers "... make open practices a pre-condition for more comprehensive review". Another example is the Transparency and Openness Promotion guidelines (TOP; Nosek et al., 2015), aimed at establishing better practices for open science. Web-based initiatives such as the Open Science Framework and AsPredicted.org provide online platforms for the preregistration of research protocols, allowing us to separate exploratory from confirmatory studies, and avoid exacerbating the file drawer problem.

Preregistration and registered reports have also been re-introduced ${ }^{1}$, advertised by proponents such as Chris Chambers with the promise of improving research quality and, by extension, trust of that research. Finally, the policies and activities of incentive structures and funding organizations are beginning to evolve, in order to accommodate the growing needs of the psychological community for transparency and accountability. For instance, calls for replication studies by high-profile journals such as the Journal of Experimental Psychology: General and the Netherlands Organisation for Scientific Research (NWO, or the 'Nederlandse Organisatie voor Wetenschappelijk Onderzoek') provide much-needed outlets, financial support and guidance for conducting and reporting replication studies, regardless of the nature of the findings.

These initiatives, while a first step in the right direction, only go so far to help the current situation because they are preemptive in nature; only prescribing best practice for the future. They cannot help untangle the messy current literature body we currently continue to build upon. The fact remains: we need replication. Therefore, psychological science needs a way to separate the wheat from the chaff; a way to determine which findings to trust and which to disregard.

When it comes to replication, however, there is a typology to speak of that separates the general concept of 'replication' out into subgroups. These different types of replication can be used for different purposes. For instance, one might be concerned with validating the theoretical framework of a phenomenon reported in an original study, or want to expand the original study methodology and design to accommodate different variables potentially of interest. In such a case, the 'conceptual' replication study is an obvious approach, where determining the theoretical importance or impact of a study or group of studies is the driving force behind the replication. On the other hand, one may seek to confirm an experimental finding under the same methodological protocol as the original article, in which case, one would conduct a direct or exact replication study. Direct replications are especially valuable in helping determine the existence of an effect. If an originally reported effect was found due to type-I error, a direct replication can be an effective tool in reinforcing results (Simons, 2014).

Conceptual and direct replications can be used in partnership to great effect to firmly establish studies. After type-I error is eliminated as the cause of significant results, via a successful direct replication ${ }^{2}$, conceptual replications can be conducted in order to establish

\footnotetext{
${ }^{1}$ We say 'reintroduced' here because many of the initiatives currently being implemented were originally suggested in concept many decades ago. For instance, in 1956, De Groot argued that relevant details of studies should be "registered in advance" (De Groot, 1956/2014).

${ }^{2}$ Nuance is needed here: Although two positive findings of the same effect both being the result of type-I
} 
the robustness and boundary conditions of an effect under different methodological protocols, and with different variables. Such a thorough and labor-intensive approach could be reserved for effects or phenomena, or groups of studies that form the foundation of entire branches of research.

The undeniable need for replications introduces a second generation of complications related to interest in conducting replication studies: a flood of new replications of existing research have reached the literature, and more are being conducted. In theory, this uptick in the number of replications being conducted is a good development for the field (especially given that up until recently, replication studies only occupied about 1 percent of the literature body: see Makel, Plucker, \& Hegarty, 2012), however in practice, so much interest in conducting replications leads to a logistical problem. There is a vast body of literature that could be subject to replication. But the question is: how does one select which studies to replicate from the ever-increasing pool of candidates out there? Which replications retread already 'well-trodden ground', and which move research forward? (Singh Chawla, 2016). These questions have serious practical implications, given the scarcity of resources (such as participants and time) in many scientific research fields.

One could say that, broadly, there are three different facets to selecting replication targets, associated with the different information contained in a published article: statistical, theoretical and methodological. First, studies can be selected for replication when their claims require additional corroboration, based on the statistical evidence reported in the publication. This is a statistical approach to determining what should be replicated first. Recall that the use of NHST is ubiquitous in psychological research- the importance of the majority of existing research findings have been quantified using $p$-values.

Although there are numerous downsides to using NHST to quantify scientific evidence (for a discussion, see Wagenmakers, 2007), we focus on one key drawback here which relates directly to our discussion. The $p$-value only allows us to reject the null hypothesis: there is a single evidence threshold, meaning that we cannot use the $p$-value to gather evidence in favor of the null hypothesis, no matter how much evidence may exist for it. Given that it is unlikely that each study reporting an effect is based on a true main effect (Ioannidis, 2005), but that studies rarely use statistical techniques to quantify evidence for the absence of an effect, there is a mismatch in what we can conclude and what we want to conclude from our statistical inference (Haucke, Misoga, Hoekstra, \& van Ravenzwaaij, 2018).

An alternative to quantifying effects in the conventional NHST framework is the Bayesian approach ${ }^{3}$. It is possible, and recommended by many prominent statisticians, to quantify the evidential strength of data via Bayes factors. So far, this possibility has found interesting applications, such as is demonstrated in the study of Hoekstra, Monden, van Ravenzwaaij and Wagenmakers (2018). Although Hoekstra and colleagues did not use Bayesian statistics for selecting replication targets, as we do in this article, their novel application of a Bayesian reanalysis could prove to be a useful method in this context (see also Pittelkow, Hoekstra, \& van Ravenzwaaij, 2018).

Bayes factors, the "... primary tool used in Bayesian inference for hypothesis testing and model selection..." (Berger, 2006), allow for the quantification of evidence in favor of

error is an unlikely scenario, it is of course possible. We suggest that a successful direct replication at least decreases the chance that an original finding was the result of type-I error.

${ }^{3}$ For a primer on this approach, please see Appendix A, taken from Field et al. (2016) 
the null hypothesis relative to the alternative, unlike the conventional $p$-value. For instance, a Bayes factor of $\mathrm{BF}_{10}=5$ means the data are 5 times more likely to have occurred under the alternative hypothesis than under the null hypothesis. In comparison, a Bayes factor of $\mathrm{BF}_{10}=1 / 5$ (or the inverse of 5 ), means the observed data are five times more likely to have occurred under the null hypothesis than under the alternative hypothesis.

When frequentist results are reanalyzed and transformed into Bayes factors, the relationship between Bayes factors and $p$-values can be strong if the analyzed studies have mostly comparable sample sizes (Wetzels et al., 2011; Aczel, Palfi, \& Szaszi, 2017). When studies have differing sample sizes, this relationship is no longer straightforward (see e.g., Hoekstra et al, 2018). Consider the following example for illustration.

We have two results of classical statistical inference:

Scenario 1: $t(198)=1.97, p=.05$

Scenario 2: $t(199998)=1.96, p=.05$

In both cases, the $p$-value is significant at the conventional alpha-level of .05, however due to the very different sample size in both scenarios, these two sets of results reflect very different levels of evidential strength. The Bayes factor, unlike the $p$-value, can differentiate between these two sets of results. Through the lens of the Bayes factor, scenario 1 presents ambiguous evidence: $\mathrm{BF}_{10}=0.94$ (i.e., the null and the alternative hypotheses are about equally likely, given the data). A Bayes factor for scenario 2 presents strong evidence in favor of the null: $\mathrm{BF}_{10}=0.03$ (i.e., the null hypothesis is about 29 times more likely than the alternative hypothesis, given the data).

In this paper, we apply a Bayesian reanalysis to several recent research findings, the end-goal being to demonstrate a technique one can use to reduce a large pool of potential replication targets to a manageable list. The Bayesian reanalysis is diagnostic in the sense that it can assist us in separating findings into three classes, or tiers of results: (1) results for which the statistical evidence pro-alternative is compelling; (2) results for which the statistical evidence pro-null is compelling; (3) results for which the statistical evidence is ambiguous. We reiterate here that, crucially, $p$-values are unable to differentiate between results which belong in the second of these categorical classes, and those that belong in the third. The third class of studies will be carried into the next 'phase' of our demonstration, wherein we further scrutinize study results with ambiguous statistical evidence on theoretical and methodological considerations that might factor into the decision to replicate.

Mackey (2012) provides some pointers on how one may select a replication target based on the theoretical content of a reported research finding. She suggests that in order to qualify as a 'candidate' for replication, a study should address theoretically important (for short, 'theoretical importance') and currently relevant research questions ('relevance'). A study also qualifies if it concerns studies in the field that are accepted as true in the field, but have yet to be sufficiently investigated ('insufficient investigation'). ${ }^{4}$ The theoretical

\footnotetext{
${ }^{4}$ We note that some of Mackey's guidelines lead to subjective decisions about what is theoretically relevant and important. What may be theoretically important in one field, may not be worth investigating in another, and so it is vital to consider the context of a potential replication target, and root one's judgments in quantifiable argumentation.
} 
approach will be explained as we describe it in a practical application later in the paper.

The last facet to selecting replication studies concerns methodological information. While many aspects of a study's methodology are highly specific to the paradigm of the article in question (e.g., the use of certain materials like visual stimuli), some elements of methodology can be discussed in general (e.g., sample size). As with the theoretical facet, methodology will be discussed in more detail during the later demonstration.

A replication study itself is beyond the scope of this paper, however we offer a demonstration of how the combined use of theory and Bayesian statistics can drive a methodical and qualitative approach to selecting replication targets in the psychological sciences. Additionally, we offer theoretical and methodological recommendations, in case such a replication were to be conducted. Please note that although the theoretical context and methodology of a study is important for selecting studies for replication, our demonstration focuses primarily on applying the Bayesian reanalysis to this challenge.

\section{Method}

We extracted statistical details from articles in the 2015 and 2016 Psychological Science and performed a Bayesian reanalysis to make a first selection of which studies could be targets for replication, based on the evidential strength of the results reported. Once this initial selection was made, we further refined the selection based on the theoretical soundness of the conclusions drawn from the selected studies, and considered the support for the finding which exists in the literature already. The approach combined quantitative and qualitative methods: on the one hand, the initial selection was based on an empirical process, and on the other, the refinement of the selection was based on a process involving judgments of the findings in the context of the literature and theory.

\section{Sample}

All Psychological Science articles from 2015 and 2016 issues were searched for reported significant statistical tests (one-sample, paired, and independent $t$-tests), associated with primary research questions. We used statistical significance as our criterion as we were interested in those results claiming the existence of an effect. All of the articles reporting $t-$ tests to test their main hypotheses used $p$-values to quantify their findings. We extracted the $t$-values and other details required for the reanalysis (including $\mathrm{N}$ and $p$-value) for 30 articles which contained $t$-tests (the data spreadsheet which logs these details for each statistic extracted is on the project's OSF page at https://doi.org/10.17605/OSF.IO/3RF8B).

Incomplete or unclear reporting practices posed a challenge in the first step of selecting which articles to reanalyze. Determining whether the executed tests were one- or two-sided was often difficult, as articles frequently failed to report the type of test conducted. Several articles which used $t$-tests as part of their main analysis strategy were ultimately not included in the reanalysis, as not all information was available (not even to the extent that we could reverse engineer other necessary details). One article, which reported two $t$-tests in support of their main finding, was excluded from the final reanalysis. Due to unclear reporting, we were unable to identify what the study's method entailed, and, therefore, how the reported results were reached. We explore the reporting problem in detail in the discussion section. 
In total, from the 24 issues of 2015/2016 Psychological Science, 326 'research articles' and 'research reports' were manually scanned for studies in which a major hypothesis was tested using a $t$-test. of these, 57 results were derived from 30 individual articles. Several articles reported more than one primary experimental finding which was analyzed using a $t$-test. Different approaches yielded judgments of whether or not a finding was of focal importance. First, if a specific finding was reported in the abstract, it would be selected (where possible). The rationale for this approach was that the abstract has only got space for documenting the most important results of the study, thus only key findings will be reported in it. A finding was also selected if somewhere in the article it was tested in a primary hypothesis, or was explicitly noted by the authors of the article as being important for the study's conclusions. Many articles reported several $t$-tests in support of a single broader hypothesis. In such cases we attempted to select the results which most directly supported the author's conclusions.

\section{Descriptive Results}

$P$-values, test statistics, sample sizes and test sidedness were collected for the purpose of the reanalysis. The $p$-values ranged in value; the largest was .047. The test statistics and sample sizes obtained also ranged greatly. The absolute test statistics ranged from 2.00 to 7.49. The range of the sample sizes is from $N=16$ to $N=484$. The distribution of study sample sizes is heavily right-skewed. The median for this sample is 54 - smaller than recent estimates of typical sample sizes in psychological research (Marszalek, Barber, Kohlhart, \& Cooper, 2011).

In the Bayesian reanalysis, we converted reported information extracted from articles into Bayes factors, to assess the strength of evidence given by each result. ${ }^{5}$ The Bayes factors ranged very widely: 0.97 to $1.9 \times 10^{10}$, or approximately 19 billion. Almost half of them are between 1 and 5 .

A clear negative relationship between the Bayes factors and the reported $p$-values is shown in Figure 1. Despite the nature of this relationship, some small $p$-values are associated with a range of Bayes factors (around the $p=.04$ mark, for instance). A positive relationship between Bayes factors and sample sizes can be seen in Figure 2. Unsurprisingly, larger sample sizes are generally associated with larger Bayes factors $(r=.71)$, though it is not the case that large sample sizes are always associated with more compelling Bayes factors. For instance, many cases in the $N=200$ region are associated with somewhat weak Bayes factors. In one case, the overall $N$ of 30 converts to a Bayes factor of over 151,000, in another case, the overall $N$ of 35 is associated with a Bayes factor of over 21,000.

\section{Quantitative Target Selection}

In this paper, we will make an initial selection based on those studies in tier 3: whose results yield only ambiguous evidence in relation to support for their reported hypotheses. For this purpose, we will judge such ambiguity, or low evidential strength, as when a study's

\footnotetext{
${ }^{5}$ Bayes factors can show evidential strength in favor of an alternative hypothesis (denoted $\mathrm{BF}_{10}$ ), or be inverted and show support for the null hypothesis (denoted $\mathrm{BF}_{01}$ ). In this article, we only discuss Bayes factors in terms of their support of the alternative, and so refrain from using the specific subscript notation or verbal indication.
} 


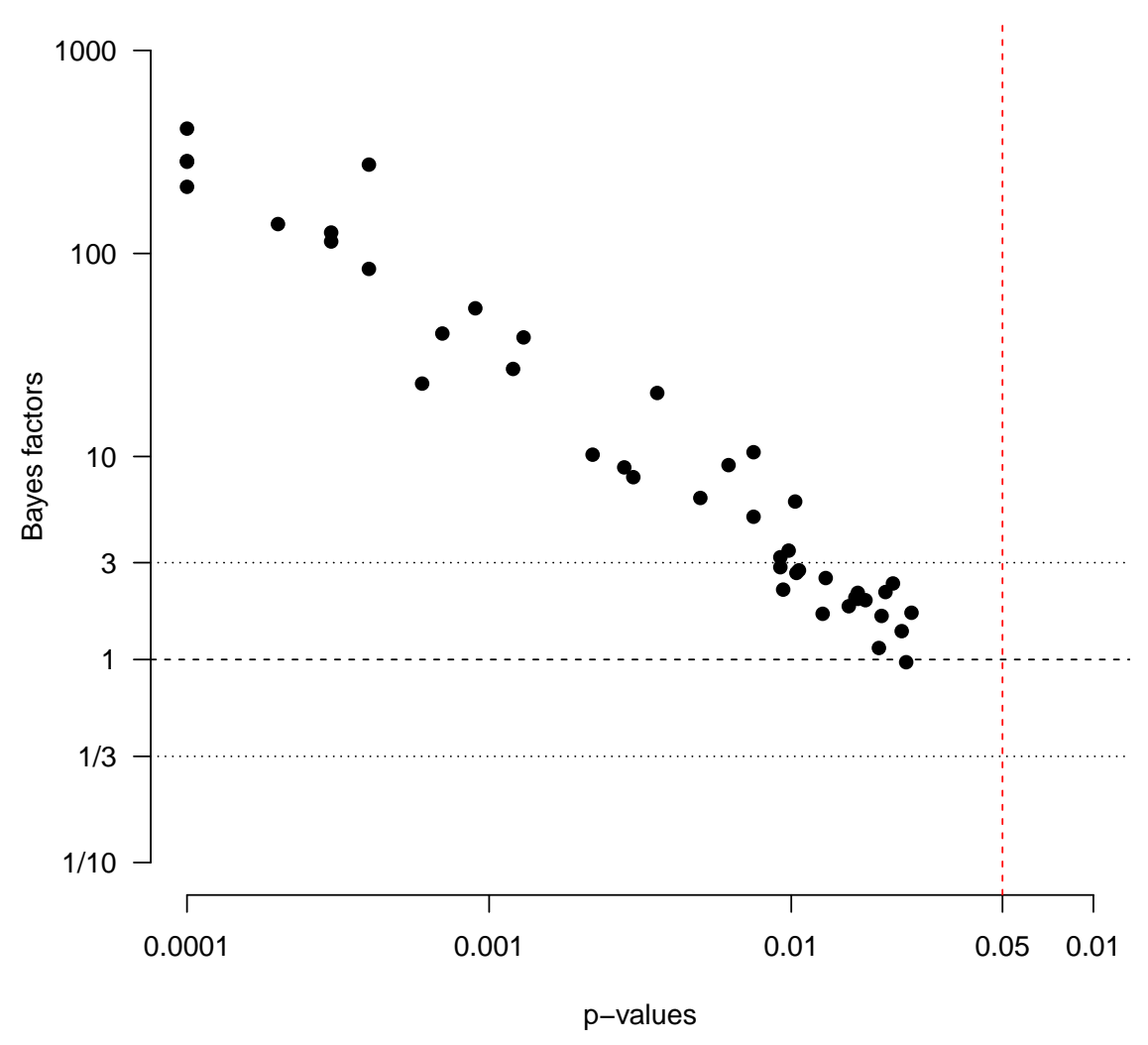

Figure 1. Scatterplot of Bayes factors and $p$-values plotted on a log-log scale. The horizontal dashed lines indicate Jeffreys' thresholds for anecdotal evidence (3, for pro-alternative cases, and the inverse for pro-null cases). The vertical red line demarcates the conventional significance level for $p$-values.

$\mathrm{BF}_{10}$ lies between $\frac{1}{3}$ and 3 , which, by Jeffrey's (1961) classification system provides no more than 'anecdotal' evidence for one hypothesis over the other.

Using the BayesFactor package in R (Morey, Rouder, \& Jamil, 2015), we calculated Bayes factors $(\mathrm{BF})$ for each test statistic using the extracted test statistics, and other information gathered: $p$-values, test statistics, sample sizes and sidedness of the test. While the vast majority did not explicitly state that they were confirmatory, most results were presented as though they were. The code written for the analysis which is associated with the data spreadsheet can be found at the project's OSF page: https://doi.org/10.17605/ OSF. IO/3RF8B.

The reanalysis revealed that the Bayesian reanalysis placed 20 results in evidence tier 3. One of these yielded a Bayes factor below 1 (0.97), which, by Jeffrey's classification system, demonstrates anecdotal pro-null evidence. The remainder of the results lie in tier 1. As we were only interested in those articles for which an effect was reported, no results falling 


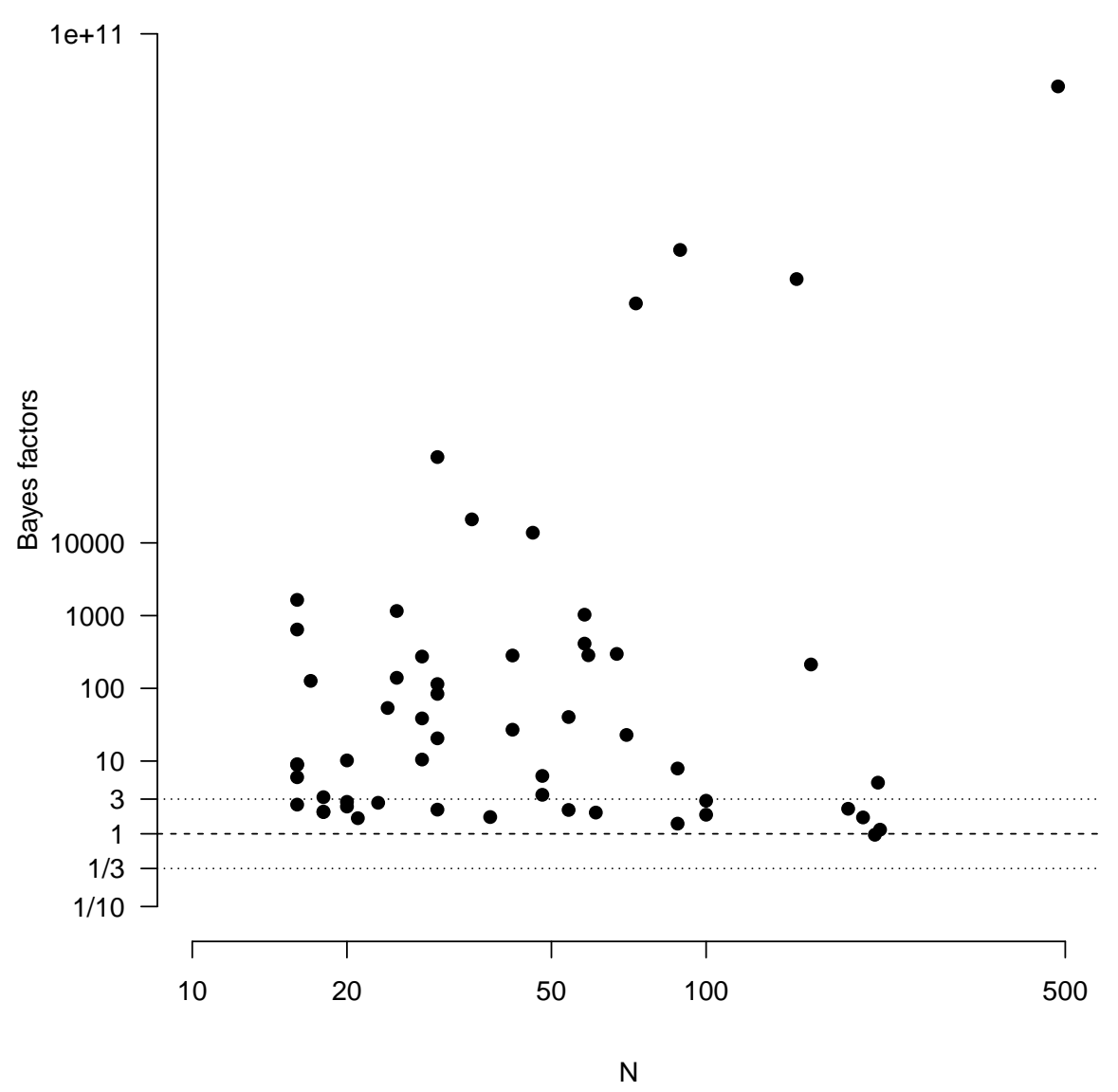

Figure 2. Scatterplot of Bayes factors and sample size plotted on a log-log scale. The horizontal dashed lines indicate Jeffreys' thresholds for anecdotal evidence (3, for pro-alternative cases; the inverse for pro-null cases). The cases in which we are interested for the reanalysis, those in tier 3, lie between the two finely dashed lines.

in tier 2 (those with compelling pro-null evidence) exist in this dataset. The reanalysis has reduced the pool of results from 57 to 20 candidates for replication. We now move onto the next stage of target selection.

\section{Qualitative Target Selection}

Of the 20 results in tier 3 , we select those demonstrating the weakest evidence for their effects. If there is an article for which many results fall in tier 3, these will also be considered. ${ }^{6}$ We will then conduct an assessment based on the qualitative criteria of Mackey (2012): theoretical importance, relevance, and insufficient investigation. Alongside Mackey's criteria, we consider the need for the finding in question to be replicated under

\footnotetext{
${ }^{6}$ We originally planned to consider those articles with the smallest Bayes factors, however, as we discuss later, there are many results with similar Bayes factors (e.g., 1.64, 1.68 and 1.70), which makes that choice alone somewhat arbitrary.
} 
different study conditions or with a different sample than the original (to establish the external validity of the effect in question), as well as replication feasibility (for instance, can this study be replicated by generally-equipped labs, or are more specific experimental set-ups necessary?). We will refer to the articles by the article number we have given them (the article and reanalysis details corresponding to these can be found in Appendix B; a full table of all the details can be found on the OSF page for this project, at https://doi.org/ 10.17605/OSF. IO/3RF8B).

The first to consider is the article revealed by the reanalysis to contain anecdotal pro-null evidence in one of its studies: article 8 . The authors of article 8 report on the socalled 'fresh start effect'. This effect refers to the use of temporal landmarks to initiate goal pursuit. More specifically, the authors' report supports claims that certain times of year (for instance, New Year's Eve) are especially potent motivators for starting new habits (such as working out, or eating more wisely). Although some evidence in this article is weakly pro-null (result 8a), one strike against naming article $8 \mathrm{a}$ as a suitable target for replication, is that the article contains a second result we reanalyzed (result 8b), that yielded a Bayes factor of 5.05 (which constitutes pro-alternative evidence).

In terms of Mackey's (2012) criteria, the study is difficult to judge as a replication target. Article 8's topic is theoretically important and certainly currently relevant: understanding the relationship between motivation and initiating healthy eating behavior is important for many reasons (for developing strategies to lowering the global burden of health due to preventable disease, for instance). However, the link between temporal landmarks and motivation has been demonstrated often and by different research groups (Peetz \& Wilson, 2013; Mogilner, Hershfield, \& Aaker, 2018; Urminsky, 2017), as well as in other studies by related groups (Dai, Milkman, \& Riis, 2014; Lee \& Dai, 2017; Dai et al., 2017).

Although this phenomenon has been the subject of many different studies, and the content of article 8 lends itself to interesting replications in which one varies, for instance, the culture of the sample, existing literature in the area already demonstrates the effect in other cultures than the USA (e.g., Germany: Peetz \& Wilson, 2013), it is not a clear replication target, in our assessment.

The majority of the remaining results in tier 3 show Bayes factors that are homogeneous in terms of their magnitude- for instance, half of the results have a Bayes factor between 1 and 2. Additionally, we see only one case in which an article with more than one reanalyzed result all fell into tier 3 (that case is article 12, containing 3 reanalyzed results). They may reflect one study of many in an article which overall, through other tests, provides strong evidence of a main effect. Both of these reasons render the majority of the sample less attractive as replication candidates.

Despite this, two articles (both featuring three low Bayes factors each) are potential targets. ${ }^{7}$ We now commit these to the qualitative assessment to determine their suitability for replication, in no particular order.

One potential replication target is article 4, in which the hypothesis that using mental imagery, or 'visualizing' can improve attention to targets in a visual search scene was tested. The authors recorded reaction times (RT) and event-related potentials (quantified as N2pc amplitudes, which reflect ongoing neural processes - in this case, attention) in

\footnotetext{
${ }^{7}$ We only target these articles to practically demonstrate how our approach can be used. We do not imply that they are of low veracity or that the results were obtained by questionable means.
} 
response to the provided stimuli. They reported support for their hypothesis: imagining the visual search for certain targets did increase the speed at which participants focused on the specified targets (indexed by the ERP), before the motor response of pressing a button to confirm they had located the target. This article yielded three $t$-tests (each testing the experimental conditions on RT), which are of interest to us. We refer to them as results 4a through 4e, respectively. They appear in the results for the first experiment, which we judged to be a clear test of their primary hypothesis. Each of these $t$-tests correspond to a small Bayes factor. The RT tests correspond to Bayes factors of 3.19, 1.99 and 2.02, while the EEG tests yielded Bayes factors of 1.83 and 2.53. (the two other $t$-tests in the sets were not significant, thus are not of interest to us for the purposes of this reanalysis).

This article meets several of the qualitative criteria too. First, the topic is theoretically important and currently relevant. Training the brain for better performance has been gaining momentum in the past decade, partly prompted by several articles that support the positive link between video-gaming and improved mental performance in different cognitive domains (such as attention: Green \& Bavelier, 2012, 2012). Exploring the link further with studies such as this can be beneficial to many areas of psychology and medicine (e.g., for working with patients of brain damage that are undergoing rehabilitation). Second, there is little supporting evidence for the link between visualization and improved attention; importantly, some of the literature aiming to reinforce the findings of article 4 contradicts it. For instance, the preregistered failed replication and extension of article 4's experiments conducted by Clarke, Barr and Hunt (2016) showed repeated searching- not visualizationimproved attention. The suitability of article 4 as a replication target is supported by fact that this article has already been a target for replication, and that that replication did not conclusively reinforce its conclusions. Other factors to consider are generalization and feasibility.

Their sample for experiment one was comprised of adults between the ages of 18 and 35 , with a gender split of $62 \%$ to $38 \%$ in favor of women. The findings of article 4 could benefit from a replication using a different sample: for instance, one with individuals from an older age range. Although age is not thought to impair neuroplasticity, older persons exhibit plasticity occurring in different regions of the brain than younger persons influencing the mechanisms underlying visual perceptual learning (Yotsumoto et al., 2014), which may influence their response to the stimuli presented in the experiments in the article. This has implications for the generalizability of the results. Another potentially important factor for consideration is gender. A recent review article by Dachtler and Fox (2017) reports clear gender differences in plasticity that are likely to influence several cognitive domains (including learning and memory), due to circulating hormones such as estrogen, which are known to influence synaptogenesis. To summarize, we find article 4 to be suitable as a replication candidate. Specifically, some of its findings could benefit from external reinforcement in the form of a conceptual replication in which factors such as age and gender are taken into consideration. Further, the results may benefit from a more in-depth exploration into the effect of searching versus visualization on attention

Another replication target that our sample yielded is article 12. Mentioned above, all reanalyzed results of article 12 (i.e., a through c) fall into tier 3. Article 12 (which includes 5 studies, each with sub-studies), explores the general hypothesis that reminders of God increase risk-taking behavior. In study 1 , which this reanalysis focused solely on (as it most 
directly tested the key hypothesis), four sub-studies are identified: 1a, 1b, 1c and 1d. The first three contain $t$-tests, while the fourth contains a chi-square test. We consider only the results of 1a through c (12a through c) for the current reanalysis.

In the study corresponding to result 12a, participants performed a priming task involving scrambled sentences. Half the participants were primed with concepts of God, by way of exposure to words such as "divine" (p. 375). The other half, which forms the control group, were exposed only to neutral words. Once participants were primed, they completed a self-report risk-taking scale which was explained to participants as being an unrelated study. This scale revealed their likely risk-taking behavior in a one to five Likert scale. In the study yielding result $12 \mathrm{~b}$, following the manipulation, participants described the likelihood that they would attempt a risky recreational task that they had described themselves at an earlier point. In the study corresponding to result $12 \mathrm{c}$, participants were tested on their interest in risk-taking via a behavioral measure, once they were primed in the first phase of the experiment. In each of these three experiments, participants primed with concepts of God reported or behaved as predicted: more predisposed to risk-taking than their neutrally-primed counterparts. Despite these three experiments yielding significant $p$-values, the reanalysis revealed three Bayes factors all suggesting the evidence is ambiguous: 1.96, 1.68 and 1.83, respectively for results 12a-c.

We now assess article 12 on the qualitative factors we described earlier. First, we consider the theoretical importance and current relevance of this article. Given that the majority of the world identifies as being religious (84\%, according to recent statistics: Hackett, Stonawski, Potančoková, Grim, \& Skirbekk, 2015), understanding the role of religion in moderating behavior is important, to say the least. According to the authors of article 12, behavior modification programs such as those employed for drug and alcohol rehabilitation use concepts of God and religion as a tool to reduce delinquent behavior. While this topic has attracted the attention of several research groups globally (meaning the article does not naturally meet the 'insufficient evidence' criterion), the reanalyzed results in article 12 go against the majority of this body of work: "... we propose that references to God can have the opposite effect, and increase the tendency to take certain types of risks" (p. 374), and do not seem to have direct strong support in the literature as yet (a paucity of indirect support can be found, e.g., Wu \& Cutright, 2018).

Study 1a features $N=61,1 \mathrm{~b} N=202$ and $1 \mathrm{c} N=100$, which corresponds to post-hoc achieved power of $.6, .63$ and .6, respectively (using the reported Cohen's $d$ values in article 12). This is relatively low power if the true effect size is that reported in article 12, and has important implications for the evidence reported in studies. Button and colleagues (2013) describe the consequences for results of low-powered designs. First, low power lowers the positive predictive value (PPV; i.e., the probability that a positive research finding reflects a real effect) when a significant effect is reported. Second, underpowered studies typically inflate the magnitude of an effect when the detected effect is true - a phenomenon dubbed the 'Winner's Curse' (Ioannidis, 2008). The Winner's Curse is thought to also affect the design and conclusions of the replication attempt: if the original effect is inflated, the replication will likely show a less impressive effect size, as the findings will, on average, converge on the true effect size (Button et al., 2013). The importance of a replication of the findings of article 12 is clear in the light of low power: as plausible as the results of article 12 may be, they are not compelling on their own simply due to low power. The considerations 
about statistical power are in line with what we have learned from our Bayesian reanalysis.

In assessing the characteristics of article 12's sample, some details indicating the suitability of article 12 for replication come to light. First, article 12 reports using Amazon's Mechanical Turk online workforce, which is comprised of approximately 80\% U.S.-based workers, and 20\% Indian workers. Given that the majority of the Mechanical Turk workers are from the U.S., and the overwhelming majority of the U.S. reports being affiliated with Christianity, we expect that the majority of this sample respond with a mindset of trusting in a God which is thought to intervene on the behalf of the faithful, responding to prayers for things like healing, guidance and help with personal troubles. The results of article 12 might be very different if the participant pool contained mostly practitioners of Buddhism (for example), as Buddhism emphasizes the importance of enlightenment (when an individual achieves an understanding of life's truth), and personal effort, rather than the intervention of a divine being (which is relevant given that feelings of security are thought to increase willingness to engage in certain behaviors: p. 374).

The age of article 12's sample is also relevant to their results, considering that the majority of workers $(>50 \%)$ were born in the 1980s. Recent polls indicate that younger individuals across Europe, the USA and Australia are less religious than their older counterparts (Harris, Chris, 2018, March 21; Wang, Amy, 2015, November 4; Schneiders, Ben, 2013, November 5), meaning that a successful replication of article 12's results with a predominantly aged population (as opposed to the mean ages of 23, 31 and 34 years, reported in the article) would demonstrate the generalizability of the finding that God-priming increases risk taking ${ }^{8}$. Another possibility also relates to age - perhaps the effect is greatly decreased in aged persons, simply by virtue of maturity: Risk-taking, even for rewards, decreases as a function of age (Rutledge et al., 2016).

Our reanalysis of article 12's results, in conjunction with other methodological and theoretical criteria considerations heavily underlines this replication candidate as a promising target, reporting results that are in need of independent corroboration. We recommend a direct, or pure replication, such that the findings exactly as they are presented can be verified. In addition, we recommend a conceptual replication in which significant changes to the characteristics of the sample are made (e.g., as mentioned, on the basis of the participants' ages and religions).

\section{Discussion}

In this paper, we performed a large scale reanalysis of the results of a selection of articles published in Psychological Science in the years of 2015 and 2016 for which primary research findings were quantified by $t$-tests. Reanalyzing these results narrowed the pool of potential replication targets from 57 to 20 candidates. The Bayes factors for these candidate studies were between 0.97 and 2.85. To further our demonstration, we selected three articles, and subjected them to the second phase of the selection process, involving qualitative assessment. The qualitative process revealed that two of these articles are suitable for replication: their findings are theoretically important and relevant, but the literature largely lacks direct corroborating evidence for the claims thus far. It revealed that the re-

\footnotetext{
${ }^{8}$ Of course, the replication as described here would need to feature different risk-taking activities, as aged persons may be averse in general to activities such as skydiving.
} 
sults could benefit from changes to the magnitude of the samples, and that several variables should be included in conceptual replications to help generalize the reported results beyond the original articles.

A set of replications for articles 4 and 12 could first determine if a true effect is likely to exist, given the results of the Bayesian reanalysis. Once an underlying true effect is found to likely exist via a direct replication, further conceptual replications could be designed to explicitly explore other cohorts to better establish the generalizability of the findings beyond the original experimental cohort. In the case of article 4 , specifically targeting participants of certain age groups may be beneficial to help determine the malleability of the effect across the lifespan. For article 12, targeting specific religious groups may assist in helping establish whether the God priming effect extends to other religions for which God is not a figure directly associated with intervention. These conceptual replications could also feature designs which vary from the originals- for instance, a replication of article 4 could feature a design in which gender is a blocking variable, or even included as a variable of interest.

Replications for both articles should contain much larger sample sizes, to help eliminate issues of reliability. In order to conduct a compelling replication study, one may need a sample size greater than that in the original study, depending on how large the sample is in the original study. Low experimental power produces some problems with reliability of original findings, leading to poor reproducibility even when other experimental and methodological conditions are ideal, which they rarely are (Button et al., 2013; Wagenmakers \& Forstman, 2014).

A simulation by Button and colleagues (2013) demonstrates an argument against the common misconception that if a replication study has a similar effect size to the original, the replication will have sufficient power to detect an effect. They show that "... a study that tries to replicate a significant effect that only barely achieved nominal statistical significance (that is, $p \sim 0.05$ ) and that uses the same sample size as the original study, will only achieve $\sim 50 \%$ power, even if the original study accurately estimated the true effect size" (p. 367). This indicates that in order to obtain sufficient power (say, $1-\beta=.8$ ) for a medium effect size in a replication study, the original sample would need to be increased by a little over double. In terms of the sample size in question, this indicates an increase from $N=105$ to $N=212$ for each of the replication studies.

Finally, some applications of our method could be constrained by the capabilities or resources of replicating labs- not all suitable replication candidates can be replicated by all interested parties, as shown in our description above. The study of article 4 is worthwhile as a replication target and warrants further investigation, however it requires specialized equipment and specific expertise to be recreated, and is therefore only feasible for select labs to seriously attempt. On the other hand, article 12 features a less specialized set of materials that could be recreated by a research group using easily-accessible university provided software (e.g., Qualtrics) and web-browsers.

Limitations of the reanalysis should be noted. It is not always clear from the reporting articles which test statistic is most suitable to extract for purposes of reanalysis. One main reason for this difficulty was outlined earlier in the methods section of the studyinconsistent reporting practices. Despite a clear and detailed article published in American Psychologist by the APA in 2008 that discusses desirable reporting standards in psychology, and other initiatives in other fields to improve research reporting (e.g., the guidelines de- 
veloped to improve the reporting of randomized-controlled trials in health-related research: Moher, Schulz, \& Altman, 2001), many researchers in the social sciences have failed to adopt them (Mayo-Wilson, 2013). To be clear, poor standards of reporting are not the norm only in psychological science. To illustrate: Mackey (2012) in linguistics research states that insufficient reporting of details important for replication is problematic in many studies (p. 26); Button and colleagues (2013) in biomedical research, discuss the relationship between insufficient reporting of statistical details and false positives in results. We also recognize that it is difficult to manage a good balance between adequate reporting and the word limit in many (especially higher-impact) journals.

Another limitation regards our reanalysis of only $t$-tests. While reanalysis of more complex designs is possible using the Bayes factor package, we only demonstrate with the simpler design of the $t$-test. We intend to show, by this demonstration, a proof of concept of a methodical and evidence-driven approach to choosing targets for replication. The Bayesian reanalysis is a clear strength, from which replicating labs can draw, however we do not advocate only the use of a Bayesian reanalysis. We must consider factors that place the article and its content in context. We must consider its appropriateness as a study for replication (is a replication feasible for less well-equipped or specialist labs?), as well as the literature body it is part of. Is the study generally well supported, or does it tell a story conflicting with existing findings? Is it theoretically important, and does it hold relevance in its current historical, social and cultural context?

A practical yet somewhat philosophical argument must be raised of how one might use the Bayesian reanalysis to prioritize replication targets. The reader critical of Bayes factors may suggest that no matter what classification one uses (Jeffreys or otherwise), Bayes factors still do not provide a complete measure of the information contained in a given original study. This reader would be right, though this can be said for any currently used quantification approach. We stress that we are not advertising the Bayesian reanalysis as the only route to a search for replication targets. We argue that it is a tool one can apply to reveal valuable information to use to distinguish between pro-null evidence and ambiguous study results. In this demonstration, it was valuable as a kind of centrifuge - filtering the studies into different 'weight' categories based on the evidence from the results, which helps us determine which studies should be replicated first. The Bayesian reanalysis can be conducted relatively easily for most interested users with the statistical software $\mathrm{R}$, using the code we have provided on our OSF page https://doi.org/10.17605/OSF.IO/3RF8B, to reduce the amount of potential replication targets, allowing individuals to direct their resources in a manner based on a justifiable and systematic method.

We would like to stress that the articles discussed in detail in this study were selected for illustration purposes only. The demonstration serves as proof of concept, and by no means aims to criticize specific studies or question their veracity. In fact, one of the three articles has two OSF badges (for more information see https://cos.io/our-services/open -science-badges-details/): one for open data, and one for open materials, indicating that the authors have made their data and study materials openly available on their project's OSF page. One of the other articles has the badge for open materials. The third article has provided access to their study materials in a supplemental folder available on the Psychological Science website. Such a commitment to transparent scientific practices are associated with research that is of higher quality, and therefore likely to be more reproducible (see the 
OSF badge page: https://cos.io/our-services/open-science-badges-details/for a discussion).

The current debate over poor reproducibility in psychology has led to a number of new ideas for how to improve our research going forward. Increased numbers of replication studies is one such advance, which has been taken up wholeheartedly by many concerned researchers. While such an initiative marks a positive and constructive move toward remedying a serious problem in our field, it is neither efficient nor useful to replicate results randomly (or worse, with unfounded accusations of poor research practice in mind). In this article, we have argued for and demonstrated an approach which is methodical and systematic, supplemented by careful and defensible qualitative analysis toward the selection of replication targets.

The approach we advocate and apply in this article can be simple and relatively fast to conduct, and affords the user access to important information about the strength of evidence contained in a published study. Although efficient, this approach has the potential to maximize the impact of the outcomes of those replications, and minimize the waste of resources that could result from a haphazard approach to replication. Combining a quantitative reanalysis with a qualitative assessment process of a large group of potential replication targets in a simple approach such as the one presented in this paper, allows the information of multiple sources to prioritize replication targets, and can assist in refining the methodology of the replication study. 


\section{Appendix A}

Explanation of the Bayes factor and Bayesian hypothesis testing

NB: The text in this section has been taken from Appendix A of Field et al. (2016), pages 91 and 92, and slightly adapted for use in this appendix.

Below we provide a short description of the Bayes factor hypothesis test; a detailed treatment is available elsewhere (Edwards, Lindman, \& Savage, 1963; Jeffreys, 1961; Ly, Verhagen, \& Wagenmakers, 2016; J. N. Rouder, Speckman, Sun, Morey, \& Iverson, 2009; J. Rouder, Morey, \& Wagenmakers, 2016; Wagenmakers, Morey, \& Lee, 2016). In addition, Etz, Gronau, Dablander, Edelsbrunner, and Baribault (2018) provide a Bayesian reading list, Mulder and Wagenmakers (2016) introduce a recent special issue on Bayes factors for psychology, and both Morey and Rouder (2015) and The JASP Team (2018) provide free and user-friendly software for obtaining Bayes factors in statistical scenarios that are common in psychological research.

Consider the case of two competing hypotheses: (1) $\mathrm{H}_{0}$, the skeptics' null hypothesis, stipulates that effect size $\delta$ equals zero, that is, $\mathrm{H}_{0}: \delta=0 ;(2) \mathrm{H}_{1}$, the alternative hypothesis, relaxes the restriction that $\delta=0$ and instead assigns it a prior distribution, allowing $\delta$ to vary, that is, $\mathrm{H}_{1}: \delta \sim f(\theta)$.

Note that both a traditional frequentist approach and a Bayesian approach refer to $\mathrm{H}_{0}$ and $\mathrm{H}_{1}$. However, only the Bayesian approach explicitly includes the alternative hypothesis when drawing statistical inference. However, the Bayesian approach requires that $\mathrm{H}_{1}$ is specified in more detail - it is not sufficient to say that $\mathrm{H}_{1}$ : delta $\neq 0$; rather, the analysts must specify a distribution for delta, as this allows $\mathrm{H}_{1}$ to make concrete predictions, the adequacy of which can be contrasted against those made by $\mathrm{H}_{0}$ (e.g., Rouder et al., in press).

An application of Bayes' rule gives:

$$
\underbrace{\frac{p\left(H_{1} \mid \text { data }\right)}{p\left(H_{0} \mid \text { data }\right)}}_{\begin{array}{c}
\text { Prior plausibility } \\
\text { about hypotheses }
\end{array}}=\underbrace{\frac{p\left(H_{1}\right)}{p\left(H_{0}\right)}}_{\begin{array}{c}
\text { Prior plausibility } \\
\text { about hypotheses }
\end{array}} \times \underbrace{\frac{p\left(\text { data } \mid H_{1}\right)}{p\left(\text { data } \mid H_{0}\right)}}_{\begin{array}{c}
\text { Predictive } \\
\text { updating factor }
\end{array}}
$$

In words, the relative posterior plausibility of $\mathrm{H}_{0}$ and $\mathrm{H}_{1}$ equals the relative prior plausibility of $\mathrm{H}_{0}$ and $\mathrm{H}_{1}$ multiplied by the relative predictive adequacy of $\mathrm{H}_{0}$ and $\mathrm{H}_{1}$ for the observed data. Equation 1 showcases three important properties of belief updating. First, the posterior plausibilities are a compromise between prior plausibilities and relative predictive performance. This means that very different prior beliefs will initially lead to divergent posterior beliefs. The only way to bring these divergent initial opinions into rough agreement is to collect data and apply a rational updating process as specified by Equation 1. We adhere to statistical tradition and focus on the predictive updating factor -the Bayes factor- that is, on the evidence that the data provide for the competing hypotheses. Proponents and skeptics may then adjust their individual beliefs accordingly.

A second property that is evident from Equation 1 is that the Bayes factor hypothesis test is symmetric in that it pits predictive performance of $\mathrm{H}_{0}$ against that of $\mathrm{H}_{1}$. Neither 
$\mathrm{H}_{0}$ nor $\mathrm{H}_{1}$ has a special status or is privileged in any way. This property allows the Bayes factor to quantify evidence in favor of the null hypothesis.

A third property is that the updating factor is solely based on relative predictive performance - the notion of a "true" model is absent. Thus, when the Bayes factor equals 4.5, this means that $\mathrm{H}_{1}$ predicted the observed data 4.5 times better than $\mathrm{H}_{0}$. This point is particularly important as it has sometimes been asserted that hypothesis tests are useless because the null hypothesis is never true (e.g., Cohen, 1994). This argument carries no force as far as the Bayes factor is concerned, as the Bayes factor is determined by out-of-sample prediction errors (Wagenmakers, Grünwald, \& Steyvers, 2006).

Thus, it may be that many effects are in the strictest sense not exactly zero. However, the effects may be so small that they drown in measurement error. As remarked by Gelman, "... when effect size is tiny and measurement error is huge, you're essentially trying to use a bathroom scale to weigh a feather - and the feather is resting loosely in the pouch of a kangaroo that is vigorously jumping up and down." (2015, April 21). A similar sentiment was expressed by Edwards et al. (p. 215-216, 1963): "Convention asks, 'Do these two programs differ at all in effectiveness?' Of course they do. Could any real difference in the programs fail to induce at least some slight difference in their effectiveness? Yet the difference in effectiveness may be negligible compared to the sensitivity of the experiment. In this way, the conventional question can be given meaning, and we shall often ask it without further explanation or apology." In the cases described above, $\mathrm{H}_{0}$ will predictively outperform $\mathrm{H}_{1}$, even though $\mathrm{H}_{0}$ may not be strictly true. As an aside, all statistical models are abstractions of reality and a case can be made that none of our models are true (J. Rouder et al., 2016). As is evident from Equation 1, this fact does not invalidate the Bayes factor.

In sum, the predictive performance of $\mathrm{H}_{0}$ and $\mathrm{H}_{1}$ is assessed by $\mathrm{p}$ (data $\mid \mathrm{H}_{0}$ : $\delta=$ 0 ) and $\mathrm{p}\left(\right.$ data $\left.\mid \mathrm{H}_{1}: \delta \sim f(\theta)\right)$, respectively. Because the null hypothesis is instantiated as a single point (i.e., $\delta=0$; for an interval specification see Morey \& Rouder, 2011), the computation of $\mathrm{p}\left(\right.$ data $\left.\mid \mathrm{H}_{0}\right)$ is straightforward. The computation of $\mathrm{p}\left(\right.$ data $\mid \mathrm{H}_{1}$ : $\left.\delta \sim f(\theta)\right)$ is only slightly more involved; for an intuitive explanation, consider $\mathrm{p}\left(\right.$ data $\left.\mid \mathrm{H}_{1}{ }^{*}: \delta=x\right)$, the prediction for a specific $\delta$ under $\mathrm{H}_{1}$. Such predictions can be computed for all possible values of $\delta$, and the overall predictive performance for $\mathrm{H}_{1}$ is then obtained by weighting the specific predictions by the prior distribution $f(\theta)$.

Mathematically, the above section can be summarized as follows:

$$
\mathrm{BF}_{10}=\frac{p\left(\text { data } \mid H_{1}\right)}{p\left(\text { data } \mid H_{0}\right)}=\frac{\int_{\Delta} p(\text { data } \mid \delta) p(\delta) \mathrm{d} \delta}{p(\text { data } \mid \delta=0)}
$$

In this equation, $\mathrm{p}(\delta)$ denotes the prior distribution $f(\theta)$. 


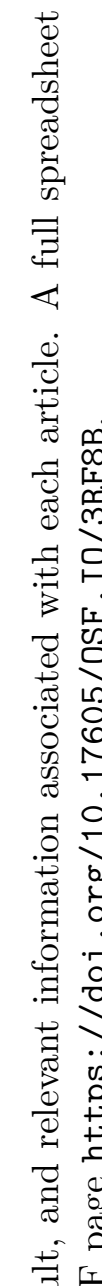

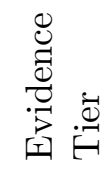$$
\text { ज्ञ }
$$ 


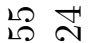

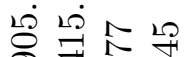

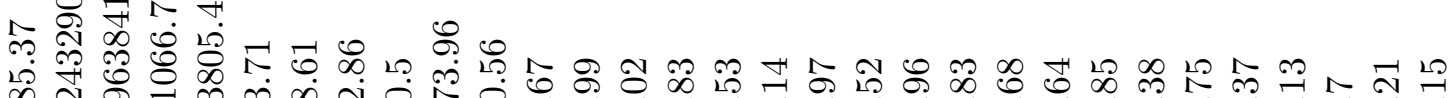

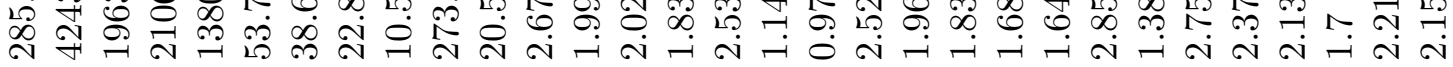

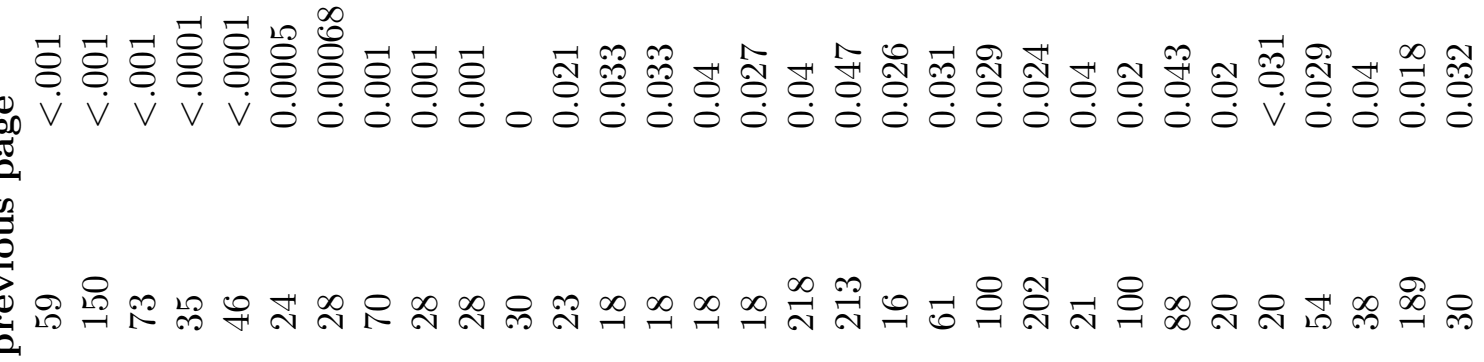

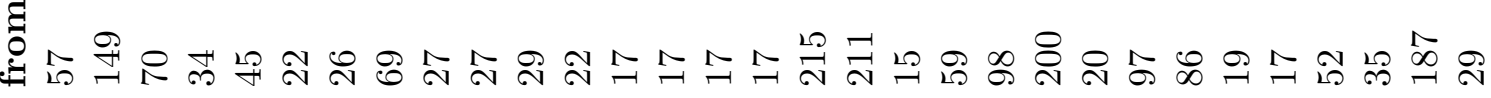
ช

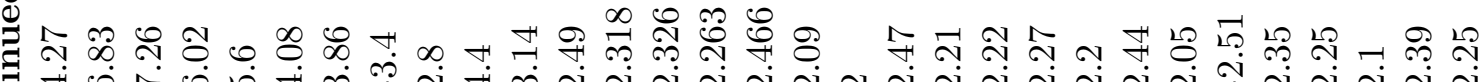
:

○ 0

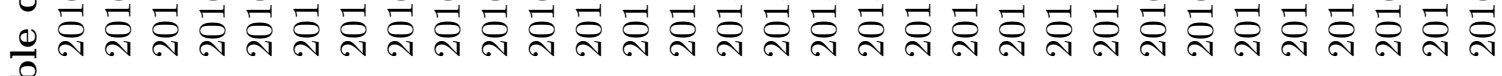
ํำ

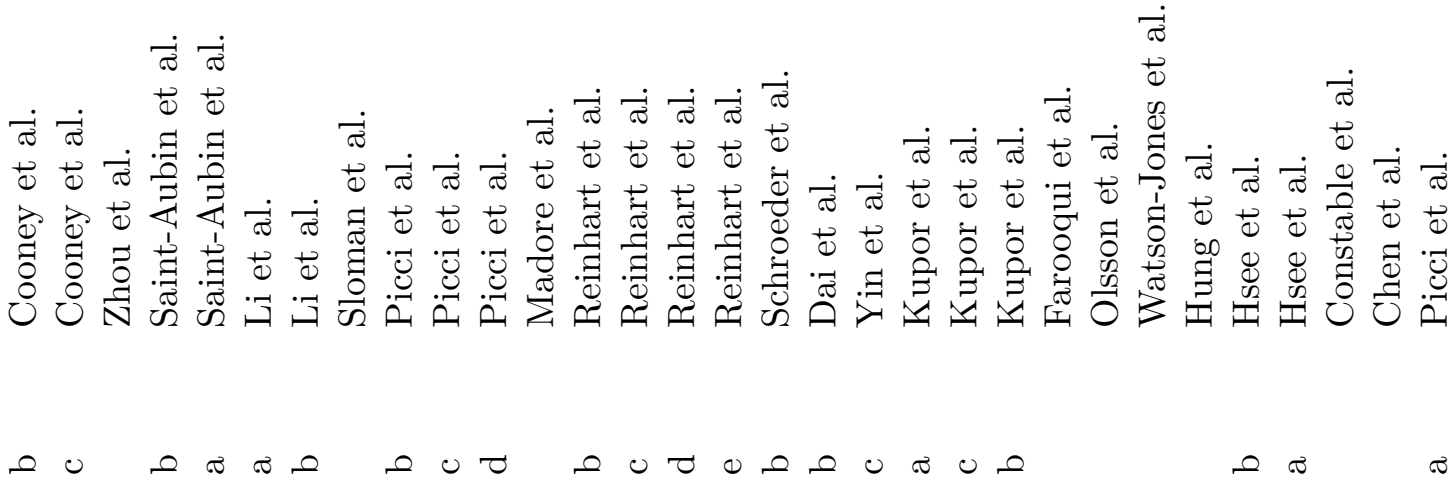

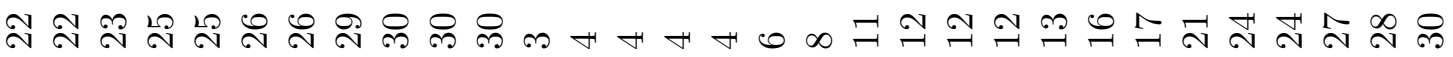




\section{Author Contributions, Competing Interests and Data Accesibility Statement}

DvR and RH conceived of the idea for the article. SMF extracted all relevant information from each article in the dataset, processed the data for the results section, and took the lead in writing the manuscript. DvR wrote the code for the Bayesian reanalysis, closely supervised the work and provided substantial feedback on the writing of the manuscript. $\mathrm{RH}$ and LFB double-checked the extractions, and provided feedback on the manuscript.

We declare no competing interests, and no external funding was used in the research for this project. All the relevant data spreadsheets and analysis scripts can be found on this project's OSF page: https://doi.org/10.17605/OSF.IO/3RF8B. 
References

Aczel, B., Palfi, B., \& Szaszi, B. (2017). Estimating the evidential value of significant results in psychological science. PLOS ONE, 12, e0182651.

Baker, M. (2016). Biotech giant publishes failures to confirm high-profile science. Nature News, 530, 141.

Begley, C. G., \& Ioannidis, J. P. (2015). Reproducibility in science: improving the standard for basic and preclinical research. Circulation Research, 116, 116-126.

Benjamin, D. J., Berger, J. O., Johannesson, M., Nosek, B. A., Wagenmakers, E.-J., Berk, R., ... others (2018). Redefine statistical significance. Nature Human Behaviour, 2, $6-10$.

Berger, J. O. (2006). Bayes factors. In S. Kotz, N. Balakrishnan, C. Read, B. Vidakovic, \& N. L. Johnson (Eds.), Encyclopedia of statistical sciences, vol. 1 (2nd ed.) (pp. 378-386). Hoboken, NJ: Wiley.

Button, K. S., Ioannidis, J. P., Mokrysz, C., Nosek, B. A., Flint, J., Robinson, E. S., \& Munafò, M. R. (2013). Power failure: why small sample size undermines the reliability of neuroscience. Nature Reviews Neuroscience, 14, 365-376.

Chang, A. C., \& Li, P. (2018). Is economics research replicable? sixty published papers from thirteen journals say âĂIJoften notâĂİ. Critical Finance Review, 7, 1-25.

Clarke, A. D., Barr, C., \& Hunt, A. R. (2016). The effect of visualization on visual search performance. Attention, Perception, $\&$ Psychophysics, 78, 2357-2362.

Cohen, J. (1994). The earth is round $(p<.05)$. American Psychologist, 49, 997-1003.

Dachtler, J., \& Fox, K. (2017). Do cortical plasticity mechanisms differ between males and females? Journal of Neuroscience Research, 95, 518-526.

Dai, H., Mao, D., Riis, J., Volpp, K. G., Relish, M. J., Lawnicki, V. F., \& Milkman, K. L. (2017). Effectiveness of medication adherence reminders tied to "fresh start" dates: A randomized clinical trial. Jama Cardiology, 2, 453-455.

Dai, H., Milkman, K. L., \& Riis, J. (2014). The fresh start effect: Temporal landmarks motivate aspirational behavior. Management Science, 60, 2563-2582.

De Groot, A. D. (1956/2014). The meaning of "significance" for different types of research. Translated and annotated by Eric-Jan Wagenmakers, Denny Borsboom, Josine Verhagen, Rogier Kievit, Marjan Bakker, Angelique Cramer, Dora Matzke, Don Mellenbergh, and Han L. J. van der Maas. Acta Psychologica, 148, 188-194.

Edwards, W., Lindman, H., \& Savage, L. J. (1963). Bayesian statistical inference for psychological research. Psychological Review, 70, 193-242.

Etz, A., Gronau, Q. F., Dablander, F., Edelsbrunner, P. A., \& Baribault, B. (2018). How to become a bayesian in eight easy steps: An annotated reading list. Psychonomic Bulletin \& Review, 25, 219-234.

Fidler, F., \& Wilcox, J. (2018). Reproducibility of scientific results. In E. N. Zalta (Ed.), The stanford encyclopedia of philosophy (Winter 2018 ed.). Metaphysics Research Lab, Stanford University. https://plato.stanford.edu/archives/win2018/entries/ scientific-reproducibility/.

Field, S. M., Wagenmakers, E.-J., Newell, B. R., Zeelenberg, R., \& van Ravenzwaaij, D. (2016). Two Bayesian tests of the GLOMO ${ }^{\text {sys }}$ Model. Journal of Experimental Psychology: General, 145, e81. 
Gelman, Andrew. (2015, April 21). The feather, the bathroom scale, and the kangaroo. Retrieved from http://andrewgelman.com/2015/04/21/feather-bathroom-scale -kangaroo/

Goodman, S. (2008). A dirty dozen: twelve p-value misconceptions. In Seminars in Hematology (Vol. 45, pp. 135-140).

Green, C. S., \& Bavelier, D. (2012). Learning, attentional control, and action video games. Current Biology, 22, 197-206.

Grimes, D. R., Bauch, C. T., \& Ioannidis, J. P. (2018). Modelling science trustworthiness under publish or perish pressure. Royal Society Open Science, 5, 171511.

Hackett, C., Stonawski, M., Potančoková, M., Grim, B. J., \& Skirbekk, V. (2015). The future size of religiously affiliated and unaffiliated populations. Demographic Research, 32, 829-842.

Harris, Chris. (2018, March 21). Young people in UK and Netherlands among Europe's least religious. Retrieved from http://www.euronews.com/2018/03/21/how-europe -s-young-adults-are-turning-their-backs-on-religion

Haucke, M., Misoga, J., Hoekstra, R., \& van Ravenzwaaij, D. (2018). Bayesian frequentists: Examining the paradox between what researchers can conclude versus what they want to conclude from statistical results. Manuscript submitted for publication.

Hoekstra, R., Monden, R., van Ravenzwaaij, D., \& Wagenmakers, E.-J. (2018). Bayesian reanalysis of null results reported in medicine: Strong yet variable evidence for the absence of treatment effects. PloS one, 13, 1-9.

Horton, R. (2015). Offline: What is medicine's 5 sigma? The Lancet, 385, 1380.

Ioannidis, J. P. (2005). Why most published research findings are false. PLoS medicine, 2, e124.

Ioannidis, J. P. (2008). Why most discovered true associations are inflated. Epidemiology, $19,640-648$.

JASP Team. (2018). JASP (Version 0.9)[Computer software]. Retrieved from https:// jasp-stats.org/

Jeffreys, H. (1961). Theory of probability. Oxford, UK: Oxford University Press.

John, L. K., Loewenstein, G., \& Prelec, D. (2012). Measuring the prevalence of questionable research practices with incentives for truth telling. Psychological Science, 23, 524-532.

Kerr, N. L. (1998). Harking: Hypothesizing after the results are known. Personality and Social Psychology Review, 2(3), 196-217.

Lee, J., \& Dai, H. (2017). The motivating effects of temporal landmarks: Evidence from the field and lab. Missouri Law Review, 82, 683-694.

Ly, A., Verhagen, J., \& Wagenmakers, E.-J. (2016). Harold Jeffreys's default bayes factor hypothesis tests: Explanation, extension, and application in psychology. Journal of Mathematical Psychology, 72, 19-32.

Mackey, A. (2012). Why (or why not), when and how to replicate research. In G. Porte (Ed.), Replication research in applied linguistics (pp. 34-69). Cambridge, UK: Cambridge University Press.

Makel, M. C., Plucker, J. A., \& Hegarty, B. (2012). Replications in psychology research: How often do they really occur? Perspectives on Psychological Science, 7, 537-542.

Marszalek, J. M., Barber, C., Kohlhart, J., \& Cooper, B. H. (2011). Sample size in psychological research over the past 30 years. Perceptual and Motor Skills, 112, 331- 
348.

Mogilner, C., Hershfield, H. E., \& Aaker, J. (2018). Rethinking time: Implications for well-being. Consumer Psychology Review, 1, 41-53.

Moher, D., Schulz, K. F., \& Altman, D. G. (2001). The consort statement: revised recommendations for improving the quality of reports of parallel group randomized trials. BMC medical research methodology, 1, 657-662.

Morey, R. D., Chambers, C. D., Etchells, P. J., Harris, C. R., Hoekstra, R., Lakens, D., ... Zwaan, R. A. (2016). The peer reviewers' openness initiative: incentivizing open research practices through peer review. Royal Society Open Science, 3, e150547.

Morey, R. D., \& Rouder, J. N. (2011). Bayes factor approaches for testing interval null hypotheses. Psychological Methods, 16, 406-419.

Morey, R. D., Rouder, J. N., \& Jamil, T. (2015). Package 'bayesFactor' [Computer software manual]. Retrieved from https://cran.r-project.org/web/packages/ BayesFactor/BayesFactor .pdf

Mulder, J., \& Wagenmakers, E.-J. (2016). Editors' introduction to the special issue "Bayes factors for testing hypotheses in psychological research: Practical relevance and new developments". Journal of Mathematical Psychology, 72, 1-5.

Nosek, B. A., Alter, G., Banks, G. C., Borsboom, D., Bowman, S., Breckler, S., . . others (2015). Promoting an open research culture. Science, 348, 1422-1425.

OSC. (2015). Estimating the reproducibility of psychological science. Science, 349, 947951.

Pashler, H., \& Wagenmakers, E.-J. (2012). Editors' introduction to the special section on replicability in psychological science a crisis of confidence? Perspectives on Psychological Science, 7, 528-530.

Peetz, J., \& Wilson, A. E. (2013). The post-birthday world: Consequences of temporal landmarks for temporal self-appraisal and motivation. Journal of Personality and Social Psychology, 104, 249-267.

Rosenthal, R. (1979). The file drawer problem and tolerance for null results. Psychological Bulletin, 86(3), 638-641.

Rouder, J., Morey, R., \& Wagenmakers, E.-J. (2016). The interplay between subjectivity, statistical practice, and psychological science. Collabra: Psychology, 2, 6.

Rouder, J. N., Speckman, P. L., Sun, D., Morey, R. D., \& Iverson, G. (2009). Bayesian ttests for accepting and rejecting the null hypothesis. Psychonomic Bulletin 8 Review, $16,225-237$.

Rutledge, R. B., Smittenaar, P., Zeidman, P., Brown, H. R., Adams, R. A., Lindenberger, U., .. Dolan, R. J. (2016). Risk taking for potential reward decreases across the lifespan. Current Biology, 26, 1634-1639.

Schneiders, Ben. (2013, November 5). Growing numbers of young Australians record no religion in census. The Sydney Morning Herald. Retrieved from https://www.smh.com.au/national/growing-numbers-of-young-australians -record-no-religion-in-census-20131124-2y3w7.html

Simons, D. J. (2014). The value of direct replication. Perspectives on Psychological Science, 9, 76-80.

Singh Chawla, D. (2016). How many replication studies are enough? Nature News, 531, 11. 
Szucs, D., \& Ioannidis, J. P. (2017). Empirical assessment of published effect sizes and power in the recent cognitive neuroscience and psychology literature. PLoS Biology, 15, e2000797.

Urminsky, O. (2017). The role of psychological connectedness to the future self in decisions over time. Current Directions in Psychological Science, 26, 34-39.

Wagenmakers, E.-J. (2007). A practical solution to the pervasive problems of $p$-values. Psychonomic Bulletin 6 Review, 14, 779-804.

Wagenmakers, E.-J., \& Forstman, B. (2014). Rewarding high-power replication research. Cortex, 51, 105-106.

Wagenmakers, E.-J., Grünwald, P., \& Steyvers, M. (2006). Accumulative prediction error and the selection of time series models. Journal of Mathematical Psychology, 50, $149-166$.

Wagenmakers, E.-J., Morey, R. D., \& Lee, M. D. (2016). Bayesian benefits for the pragmatic researcher. Current Directions in Psychological Science, 25, 169-176.

Wang, Amy. (2015, November 4). Today's young Americans are less religious - and a lot more likely to stay that way. Retrieved from https://qz.com/540395/todays-young -americans-are-less-religious-and-a-lot-more-likely-to-stay-that-way/

Wetzels, R., Matzke, D., Michael, M. D., Rouder, J. N., Iverson, G. J., \& Wagenmakers, E.J. (2011). Statistical evidence in experimental psychology: An empirical comparison using $855 t$ tests. Perspectives on Psychological Science, 6, 291-298.

Woolston, C. (2015). Online debate erupts to ask: is science broken? Nature News, 519, 393.

Woolston, C. (2016, March 24). Failure is moving science forward. FivethirtyEight. Retrieved from https://fivethirtyeight.com/features/failure-is -moving-science-forward/

Wu, E. C., \& Cutright, K. M. (2018). In god's hands: How reminders of god dampen the effectiveness of fear appeals. Journal of Marketing Research, 55, 119-131.

Yotsumoto, Y., Chang, L.-H., Ni, R., Pierce, R., Andersen, G. J., Watanabe, T., \& Sasaki, Y. (2014). White matter in the older brain is more plastic than in the younger brain. Nature Communications, 5, 5504. 